\title{
Multiseptate Gallbladder in a Patient with Biliary Dyskinesia
}

\author{
Michael Talalaev ${ }^{1}$ Ralph Tawil ${ }^{1}$ Humberto Rios ${ }^{1} \quad$ Jose Rey ${ }^{1}$ Huma Zaman ${ }^{2}$ Samantha Blain-Maiza \\ ${ }^{1}$ Department of Internal Medicine, Larkin Community Hospital, \\ Miami, Florida, United States \\ 2Department of Diagnostic Radiology, Larkin Community Hospital, \\ Miami, Florida, United States

\begin{abstract}
Address for correspondence Ralph Tawil, DO, Department of Internal Medicine, Larkin Community Hospital Palm Springs Campus, 1475 W 49 ${ }^{\text {th }}$ PI, Hialeah, FL 33012, United States (e-mail: rtawil@larkinhospital.com).
\end{abstract}

J Gastrointestinal Abdominal Radiol ISGAR:2020;3(suppl S1):S77-S79

\begin{abstract}
Keywords

- multiseptate gallbladder

- biliary dyskinesia

- biliary colic

- congenital malformation

Biliary dyskinesia due to a multiseptate gallbladder has been rarely described in literature. Septations within the lumen of the gallbladder can lead to bile resistance, causing symptoms of abdominal pain, nausea, and vomiting. The majority of cases are due to congenital malformation during the development of the gallbladder. Ultrasound (US) is usually sufficient to make the diagnosis, although other imaging techniques can be used for confirmation. We present the case of a 60 -year-old female who presented to the emergency room (ER) with biliary pain due to septations in the lumen of her gallbladder.
\end{abstract}

\section{Introduction}

Evaluation of the older patient with abdominal pain can present as a unique challenge to the emergency room (ER) physician. Abdominal pain is a common complaint in the emergency departments, comprising approximately $5 \%$ of total visits. ${ }^{1}$ Although rare, abdominal pain can sometimes be caused by anatomical or congenital malformations. In our article, we present and describe the case of acute abdominal pain due to a multiseptate gallbladder. A multiseptate gallbladder is characterized by multiple thin septa within the gallbladder lumen, creating different sized chambers. First described in 1963 by Simon and Tandon, ${ }^{2}$ the multiseptate gallbladder has been described as one of the rarest congenital malformations of the gallbladder. Septa have been reported to be single or multiple. Some common presenting features include right upper quadrant (RUQ) abdominal pain, nausea, and vomiting; however, the patient can sometimes present as asymptomatic in which imaging studies will make the discovery. The cause appears to be congenital; nonetheless, a few mechanisms have been postulated.

\section{Case Report}

A 60-year-old female presented to the ER with a complaint of abdominal pain. The patient reported pain located in the

DOI https://doi.org/ 10.1055/s-0040-1715537 ISSN 2581-9933.
RUQ radiating to the right flank. The pain was described as sharp and stabbing, rated at 10/10, and associated with nausea and vomiting. Symptoms were worsened by movement with no alleviating factors. Past medical history of the patient included arthritis, asthma, insulin-dependent diabetes mellitus (IDDM), gout, hyperlipidemia, hypertension, hypothyroidism, and seizures. Vital signs on arrival to the ED were BP of $131 / 64$, pulse of 94 , respiratory rate of 20 , and temperature of 98.9 . On physical examination, the abdomen was nondistended with normal bowel sounds. Upon palpation, tenderness was noted in the right upper and lower quadrant with voluntary guarding. There was no rebound tenderness; however, Murphy's sign was present. Labs ordered showed amylase and lipase within normal limits and white blood cell count within the normal range. RUQ ultrasound (US) showed septations within the gallbladder lumen that divided the gallbladder into few chambers. This was viewed precisely in the transverse and sagittal plane using the GE Vivid S70 Ultrasound Machine, manufactured in the USA ( $\boldsymbol{-}$ Figs. 1 and 2). Furthermore, the gallbladder was normal in size with normal wall thickness. US showed no pericholecystic fluid with normal sonolucency and no signs of gallstones. With these findings, biliary dyskinesia due to multiseptate gallbladder was diagnosed. The patient received IV fluids, ketorolac, 


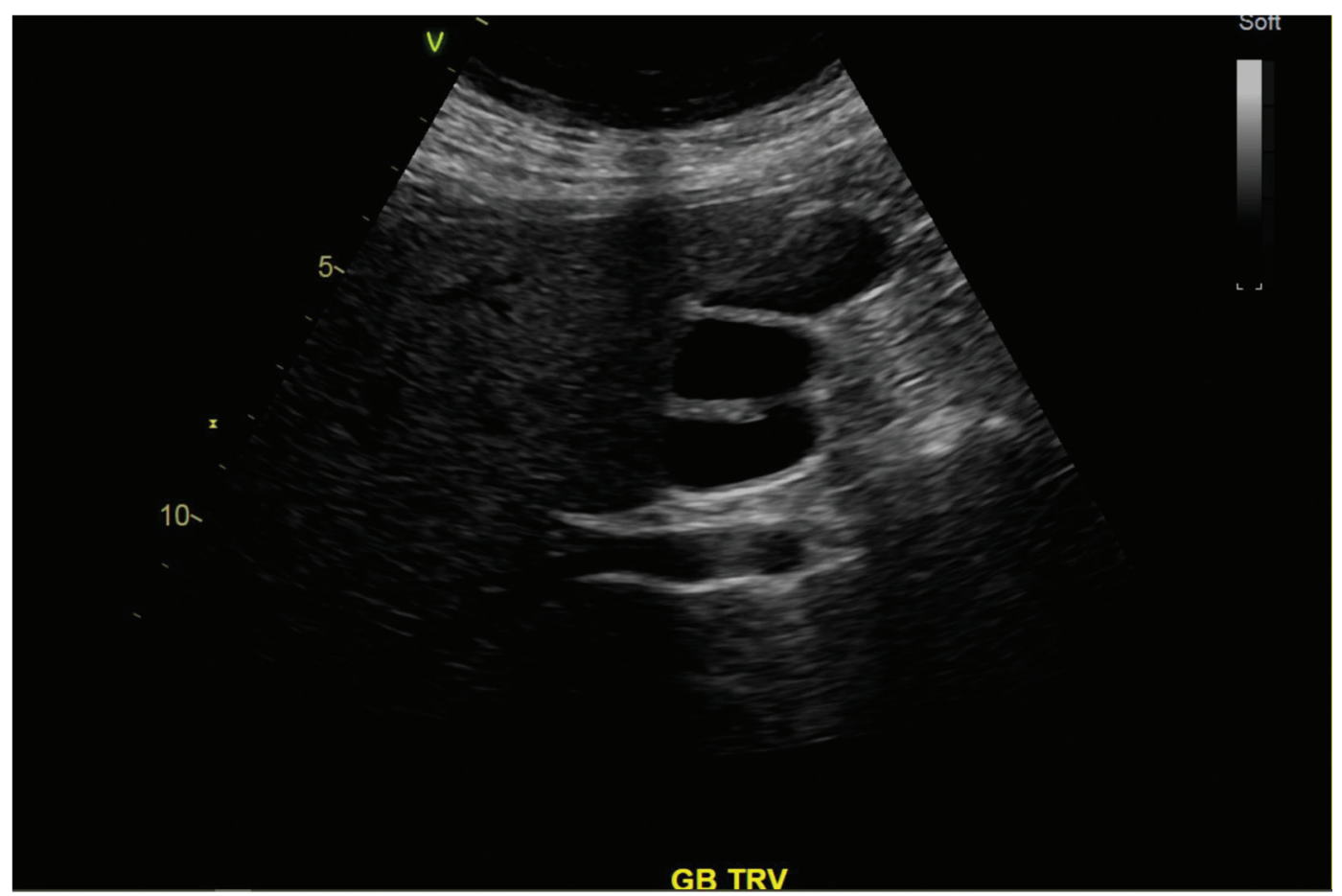

Fig. 1 Gallbladder ultrasound showing septations in the transverse plane.

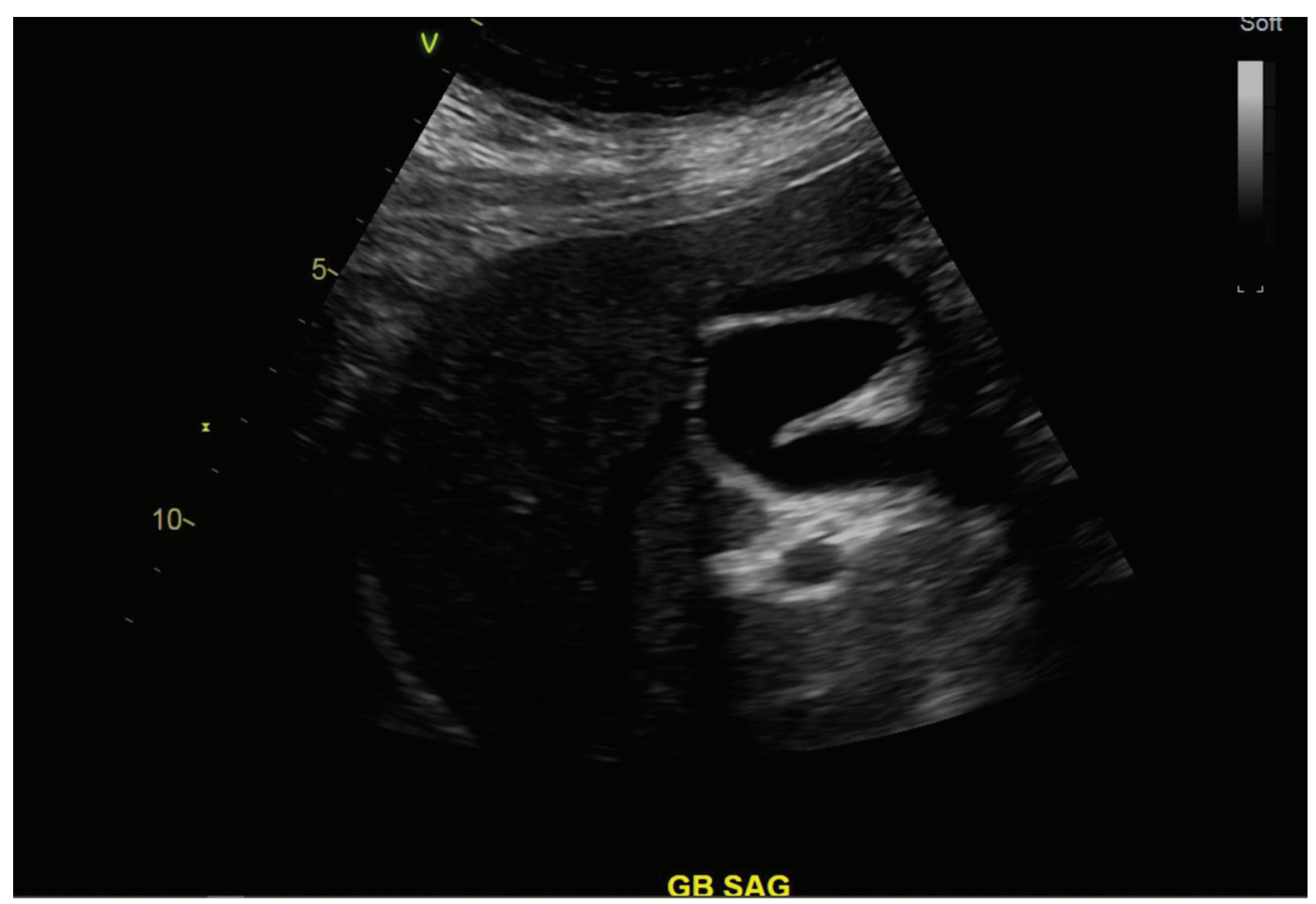

Fig. 2 Gallbladder ultrasound showing septations in the sagittal plane.

and morphine for pain control. Her condition subsequently improved, and she was discharged home for outpatient follow-up. No upper endoscopy was performed.

\section{Discussion}

Undifferentiated abdominal pain remains the diagnosis for approximately $25 \%$ of patients discharged from the ED and between 35 to $41 \%$ of those who are admitted to the hospital. ${ }^{4-7}$ The reported incidence of gallbladder anomalies is only $0.1 \% .^{8}$ In our report, we presented the case of abdominal pain caused by a rare congenital malformation of the gallbladder. The congenital malformations of the gallbladder are classified as anomalous forms, abnormal position, and absence. ${ }^{9}$ The multiseptate gallbladder is a type of rare anomaly form, first described by Simon and Tandon in 1963. ${ }^{2}$ The referred patient presented to the ER 
with severe biliary-like abdominal pain and was found to have a multiseptate gallbladder on US imaging. Septations in the gallbladder form due to incomplete vacuolization of the cystic bud during development or excessive "wrinkling" of the wall of the gallbladder. ${ }^{10,11}$ The small cavities in a multiseptate gallbladder communicate freely with each other, but the thick and viscid bile encounters resistance to passage through small openings of the septa. ${ }^{12}$ It is due to this reason that the multiseptate gallbladder can cause abdominal pain in patients. While there have been reports that described asymptomatic cases, ${ }^{13}$ most patients still present with acute abdominal symptoms such as RUQ pain, nausea, vomiting, anorexia, fever, and miscellaneous gastrointestinal symptoms. In our case, it appears that the patient's gallbladder had septa primarily responsible for the biliary dyskinesia and abdominal pain. RUQ ultrasound of the gallbladder is usually sufficient to establish diagnosis, although other imaging such as $\mathrm{CT}$, magnetic resonance cholangiopancreatography (MRCP), and endoscopic retrograde cholangiopancreatography (ERCP) have been utilized in a few cases. The essential diagnostic features are multiple fine, nonshadowing septa that bridge the gallbladder lumen. ${ }^{13}$ The pattern of septa may significantly vary from one case to another. While the septa may present in the entire gallbladder, there have been some cases where the septa were confined only to the neck, body, or fundus. Our case presented multiple thin septa throughout the lumen of the gallbladder using an US machine.

\section{Conclusion}

In conclusion, the multiseptate gallbladder is a rare cause of abdominal pain in the ED. We presented a case where severe abdominal pain was caused by septa within the body of a gallbladder. Most reported cases have described a congenital cause as a possible explanation of this finding, usually due to incomplete vacuolization of the cystic bud. Abdominal pain arises due to bile encountering resistance, as it passes through the septa causing distension and pressure over the gallbladder walls. Cases usually present with severe abdominal pain, nausea, and vomiting, and US is considered to be the most sufficient modality to make the diagnosis.

\section{Conflict of Interest}

None declared.

\section{References}

1 Kamin RA, Nowicki TA, Courtney DS, Powers RD. Pearls and pitfalls in the emergency department evaluation of abdominal pain. Emerg Med Clin North Am 2003;21(1):61-72, vi

2 Simon M, Tandon BN. Multiseptate gallbladder. A case report. Radiology 1963;80:84-86

3 Deutsch AA, Englestein D, Cohen M, Kunichevsky M, Reiss R. Septum of the gallbladder, clinical implications and treatment. Postgrad Med J 1986;62(728):453-456

4 Powers RD, Guertler AT. Abdominal pain in the ED: stability and change over 20 years. Am J Emerg Med 1995;13(3):301-303

5 Irvin TT. Abdominal pain: a surgical audit of 1190 emergency admissions. Br J Surg 1989;76(11):1121-1125

6 Jess P, Bjerregaard B, Brynitz S, et al. Prognosis of acute nonspecific abdominal pain. A prospective study. Am J Surg 1982;144(3):338-340

7 Lukens TW, Emerman C, Effron D. The natural history and clinical findings in undifferentiated abdominal pain. Ann Emerg Med 1993;22(4):690-696

8 Johnson SR, Development of the liver and biliary apparatus. In: Romanes GJ, ed. Cunninghams Textbook of Anatomy. 12th ed. Oxford, Oxford University Press. 1987;485

9 Yamamoto T, Matsumoto J, Hashiguchi S, Yamaguchi A, Sakoda K, Taki C. Multiseptate gallbladder with anomalous pancreaticobiliary ductal union: a case report. World J Gastroenterol 2005;11(38):6066-6068

10 Haslam RH, Gayler BW, Ebert PA. Multiseptate gallbladder. A cause of recurrent abdominal pain in childhood. Am J Dis Child 1966;112(6):600-603

11 Bhagavan BS, Amin PB, Land AS, Weinberg T. Multiseptate gallbladder. Embryogenetic hypotheses. Arch Pathol 1970;89(4): 382-385

12 Strauss S, Starinsky R, Alon Z. Partial multiseptate gallbladder: sonographic appearance. J Ultrasound Med 1993;12(4): 201-203

13 Lev-Toaff AS, Friedman AC, Rindsberg SN, Caroline DF, Maurer AH, Radecki PD. Multiseptate gallbladder: incidental diagnosis on sonography. AJR Am J Roentgenol 1987;148(6): $1119-1120$ 